\title{
Localisation exceptionnelle d'une tumeur glomique au niveau du palais mou
}

\author{
Suzy Flore Assini Eyogho-Ongala1,2, Alain Patrick Makungư ${ }^{2}$, Jean Claude Ekogha Ekogha², Sidonie Nguizi Ogoula ${ }^{3}$, Soukèye Dia Tine ${ }^{4}$ \\ ${ }^{1}$ Service de stomatologie et chirurgie maxillo-faciale. HIAOBO Libreville, Gabon \\ 2 Service de stomatologie et chirurgie maxillo-faciale. Centre hospitalier de Libreville, Gabon \\ ${ }^{3}$ Service d'anatomopathologie de la faculté de médecine de Libreville, Gabon \\ ${ }^{4}$ Service de chirurgie buccale, département d'odontologie UCAD, Dakar, Sénégal \\ Correspondance : eyoghoass@yahoo.fr
}

(Reçu 21 février 2015, accepté le 22 mai 2015)

Mots clés :

tumeur glomique / palais mou / diagnostic / traitement

Key words:

glomus tumor / soft palate / diagnosis / treatment
Résumé - Observation : M. G.R. âgé de 30 ans avait consulté pour une tumeur du voile du palais. La tumeur, ferme, d'environ $5 \mathrm{~cm}$, était ulcérée. L'aspect clinique et morphologique évoquait une tumeur de type malin. La tomodensitométrie maxillo-faciale avait objectivé une tumeur maligne du palais devant un processus très expansif. L'examen histologique avait conclu à un glomangiome. Le traitement a été chirurgical. Discussion : la tumeur glomique est une tumeur bénigne des tissus mous de localisation habituelle unguéale et de la face palmaire de la dernière phalange. La localisation palatine est exceptionnelle et fait discuter d'autres diagnostics cliniques. L'imagerie n'est qu'une aide au diagnostic. Le diagnostic est anatomopathologique et le traitement chirurgical consiste en une exérèse large évitant toute récidive qui reste très rare.

\begin{abstract}
Case report: Mr GR, aged 30, consulted for a soft palate tumor. It was a firm ulcerated tumor, about 5 $\mathrm{cm}$ in size. The clinical and morphological diagnosis suggested a malignant tumor. The maxillo-facial CT scan showed a malignant tumor of the palate before a very expansive process. The histological examination revealed a glomangioma, and the treatment was surgical. Discussion: A glomus tumor is a benign tumor of soft tissue usually localized on the nail and the palmar surface of the distal phalange. This rare palatal localization involves a discussion of other clinical diagnoses. Radiology is of diagnostic help. Diagnosis is anatomopathological and treatment is surgical, consisting of a wide excision, avoiding recurrences, which are very rare.
\end{abstract}

\section{Observation}

M. G.R., âgé de 30 ans, sans antécédent particulier, avait consulté au service de chirurgie maxillo-faciale pour une tuméfaction du palais mou. À l'interrogatoire, on retrouvait une notion de dysphagie des aliments solides accompagnée d'une évolution progressive de la tumeur depuis cinq mois dans un contexte non fébrile et sans douleur. L'examen clinique retrouvait un sujet en bon état général, une tuméfaction du voile du palais d'environ $5 \mathrm{~cm}$ dans son grand axe, surmontée latéralement d'une zone nécrotique, de consistance ferme et ne saignant pas au contact mais obstruant le carrefour aérodigestif (Fig. 1). Cette tuméfaction s'accompagnait d'un nasonnement lors de l'élocution. Le diagnostic suspecté était celui d'une tumeur maligne du palais mou, devant son caractère évolutif. La tomodensitométrie maxillo-faciale semblait confirmer ce diagnostic en montrant des images d'un processus expansif tissulaire du palais, hétérogène mesurant $36 \times 33$ mm, étendu sur une hauteur de $6 \mathrm{~cm}$. Ce processus avait pour point de départ le muscle du voile du palais droit et s'étendait en haut au contact du palais osseux de la voute; en bas, il faisait protrusion dans le rhinopharynx, en avant il était au contact de l'os vomer et du cornet inférieur gauche, latéralement il envahissait les muscles péristaphyliens externe et interne droits (Fig. 2). Devant la symptomatologie, la décision d'une exérèse chirurgicale large avait été prise et réalisée. L'étude histopathologique de la pièce opératoire révélait une tumeur glomique sans signe de malignité à partir des éléments histologiques et immunohistochimiques suivants : on notait d'une part sur la coloration à l'hématéine-éosine (HE) une prolifération monotone des petites cellules rondes ou polygonales à cytoplasme abondant et mal limité (Fig. 3) ; d'autre part, un marquage nucléaire de l'anticorps protéine S100 était positif (Fig. 4). Une réaction positive des marqueurs AML était retrouvée (Fig. 5) ainsi qu'une réaction CD34 cytoplasmique positive des cellules endothéliales (Fig. 6). Les réactions au 


\section{A S C L I N I Q U E}

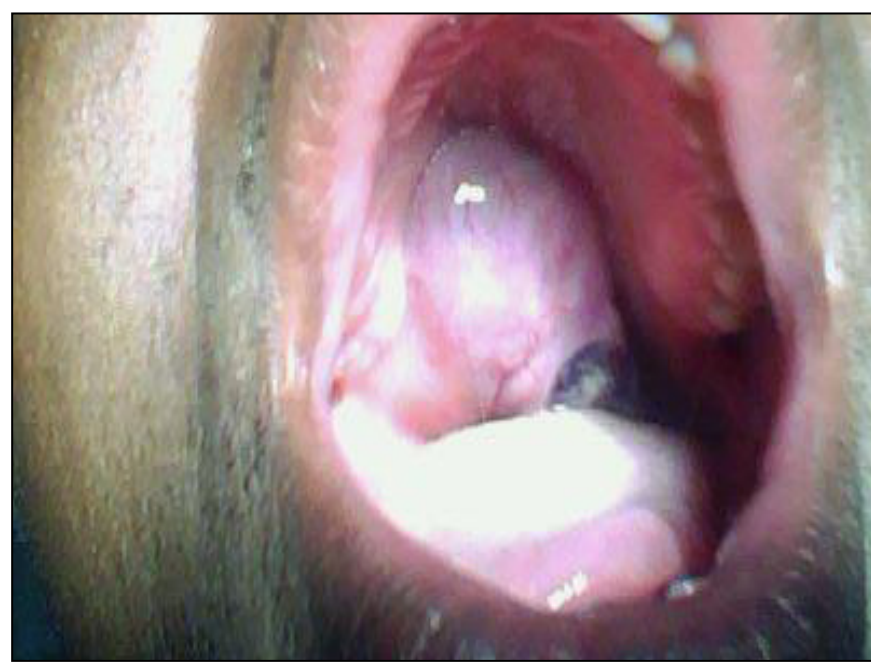

Fig. 1. Vue macroscopique bouche ouverte montrant la masse tumorale du voile du palais à gauche avec une zone nécrotique postérieure. Fig. 1. Macroscopic view mouth opened showing left soft palate tumoral mass and necrotic back region.

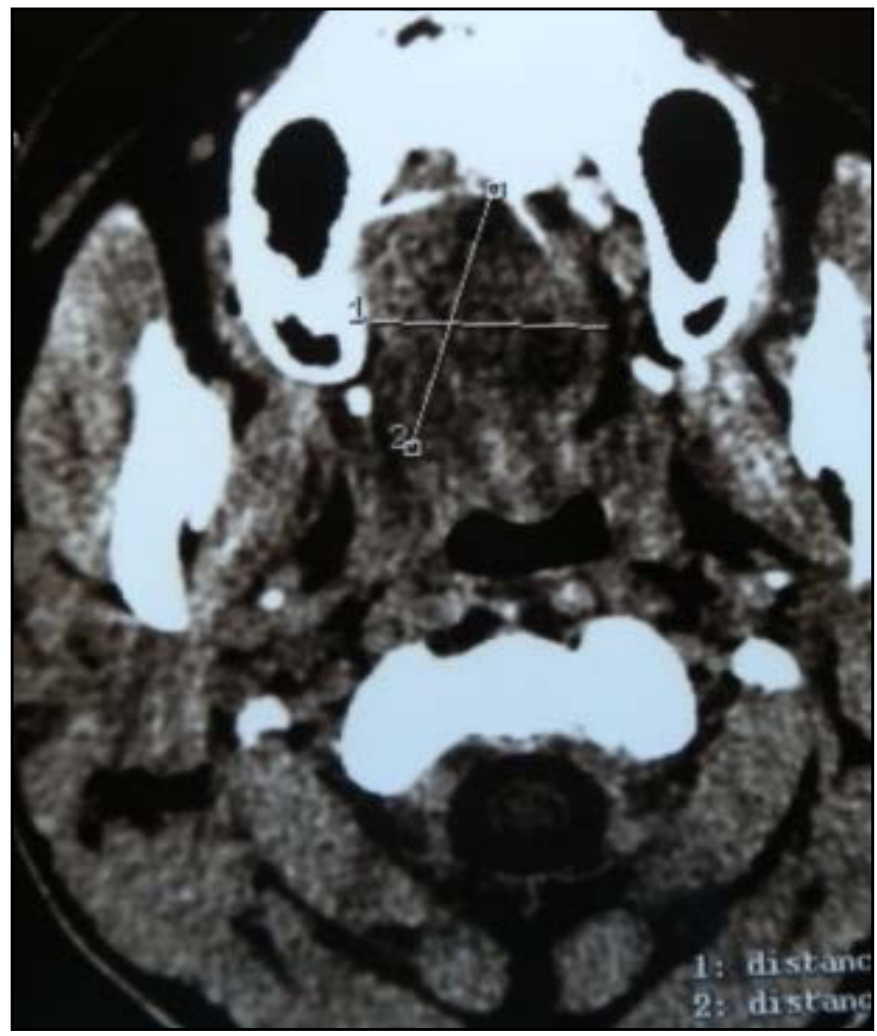

Fig. 2. CT-scan axial montrant le processus expansif du voile du palais.

Fig. 2. Axial CT-scan showing the soft palace expansive process.

marqueur Ki67 et à la desmine étaient négatives. Cette tumeur était donc classée de type glomangiome. Les suites opératoires étaient simples avec une diminution du nasonnement.

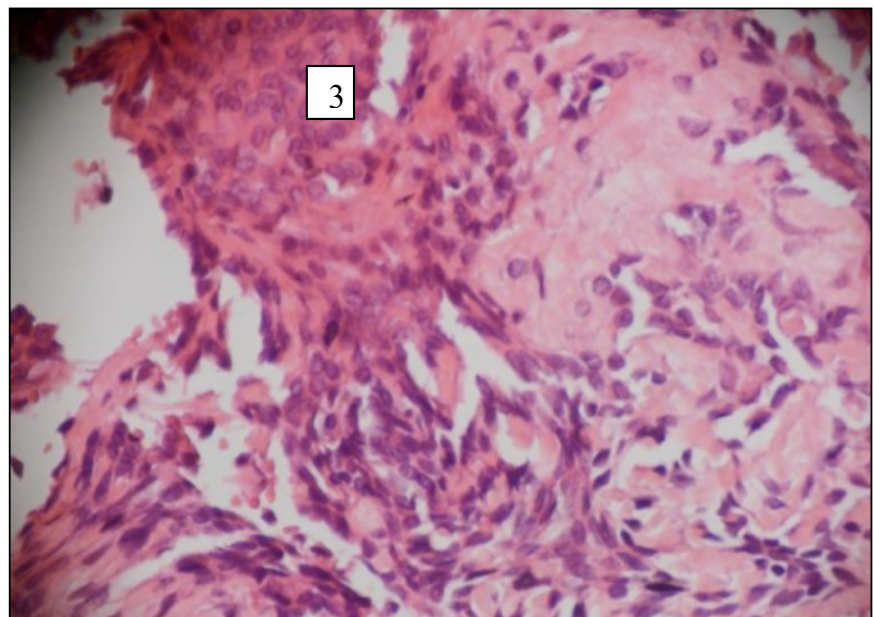

Fig. 3. Prolifération monotone de petites cellules rondes ou polygonales à cytoplasme abondant et mal limité. Le noyau est rond sans atypie; présence de plusieurs lumières.

Fig. 3. Small round and poligonal cells monotonus poliferation with plentiful cytoplasm and badly border. The core is round and typical; several chinks.

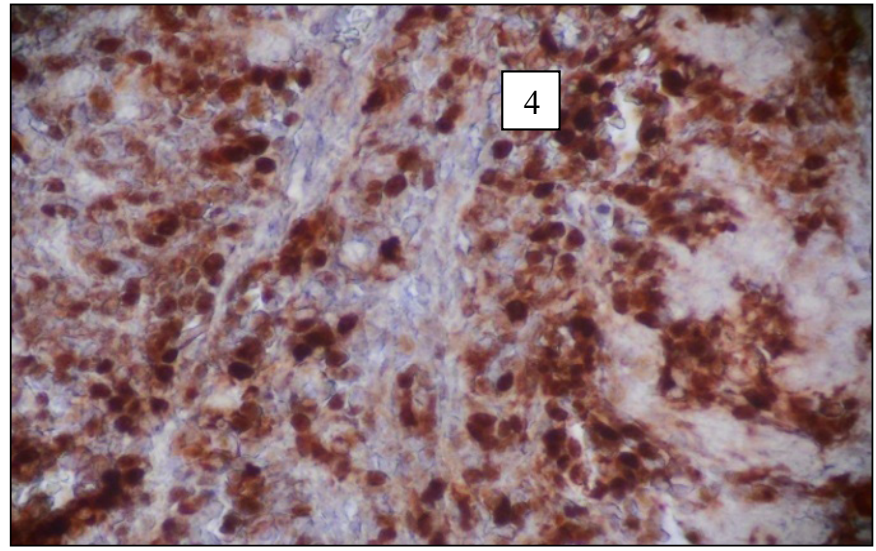

Fig. 4. Réaction nucléaire positive à la protéine S100. Le marquage apparaît nucléaire et cytoplasmique.

Fig. 4. Positive nuclear reaction for S100 protein. Labeling appeared nuclear and cytoplasmic in tumor cells.

\section{Discussion}

Les tumeurs glomiques sont des tumeurs bénignes dues à une augmentation de volume des globus ou hamartomes de l'appareil neuromyoartériel. Ce sont des tumeurs vasculaires à localisation exceptionnelle dans la cavité nasale, la trachée, les poumons et l'estomac [1-3], mais préférentielle au niveau du derme profond, au niveau de la région sous-cutanée des membres et plus précisément à la région sous-unguéale des doigts où elles peuvent éroder la dernière phalange puis le reste du membre [4-6]. 


\section{A S C L I N I Q U E}

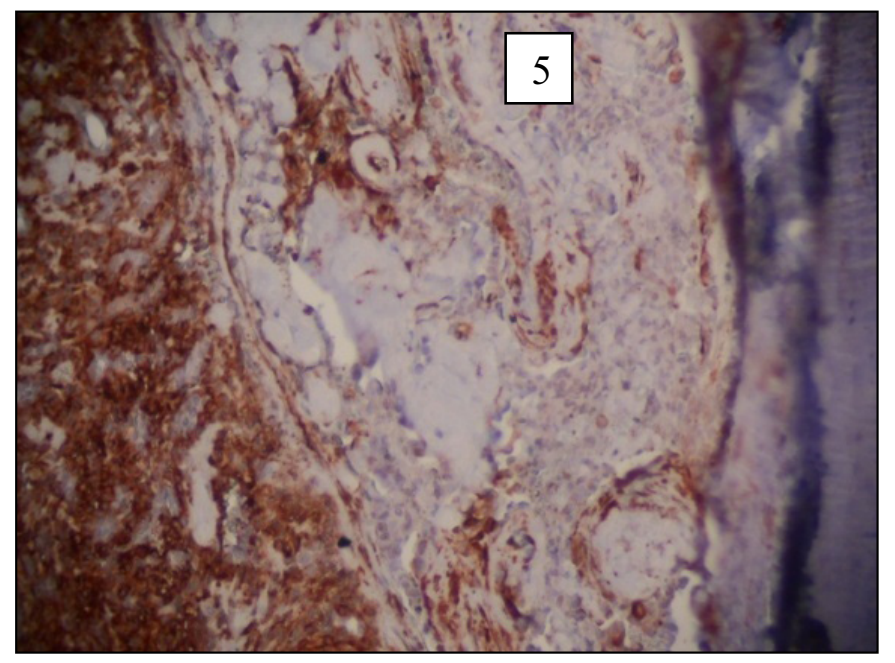

Fig. 5. Réaction positive des marqueurs AML au niveau des cellules tumorales.

Fig. 5. AML positive reaction in tumor cells.

La tumeur glomique a été décrite pour la première fois en 1812 par Wood sous la forme de « nodules sous-cutanés douloureux », mais c'est Masson qui, en 1927, dans une étude à propos de trois cas au niveau de la main, en a précisé l'origine et lui a donné le nom de tumeur glomique [7]. Il s'agit d'une tumeur bénigne vasculaire due à une augmentation de volume des globus neuro-myo-artériels à localisation exceptionnelle otorhinolaryngologique [8]. Cette tumeur s'observe surtout chez l'adulte ayant un âge variant entre 30 et 50 ans, ce qui correspondait à ce patient, mais elle est plus fréquente chez la femme, de l'ordre de $75 \%$ [4-6]. Son siège de prédilection se situe au niveau des membres et surtout leurs extrémités et, dans ces cas, elle est de petite taille, de l'ordre de $3 \mathrm{~mm}$. Lorsqu'elle siège sur d'autres organes, sa taille peut varier de 0,2 à $5 \mathrm{~cm}$ [4]. Dans le cas présent, elle était de l'ordre de $5 \mathrm{~cm}$. La symptomatologie est bien codifiée dans sa localisation unguéale, même si le diagnostic clinique est souvent difficile et peut être retardé jusqu'à 5 ans [9] d'où la triade clinique décrite par Belkourchia et al. [4] en 2005 : douleur paroxystique, sensibilité au froid et nodule tumoral ; cette notion de douleur souvent exacerbée par un changement de température est retrouvée par Theumann et al. en 2005 [10] ; ce qui n'existe pas dans les autres localisations. Dans cette observation, on notait seulement la présence d'un nodule tumoral. Les caractères cliniques de cette tumeur sont tels que le diagnostic peut être erroné. Ainsi, on peut la confondre avec un hématome lorsqu'elle se présente sous la forme d'une tuméfaction de coloration bleuâtre, un hémangiome ou un carcinome adénoïde kystique dans sa localisation palatine. L'orientation diagnostique est alors difficile, vu la symptomatologie atypique que le bilan morphologique, surtout la tomodensitométrie, objective. Cette dernière présente

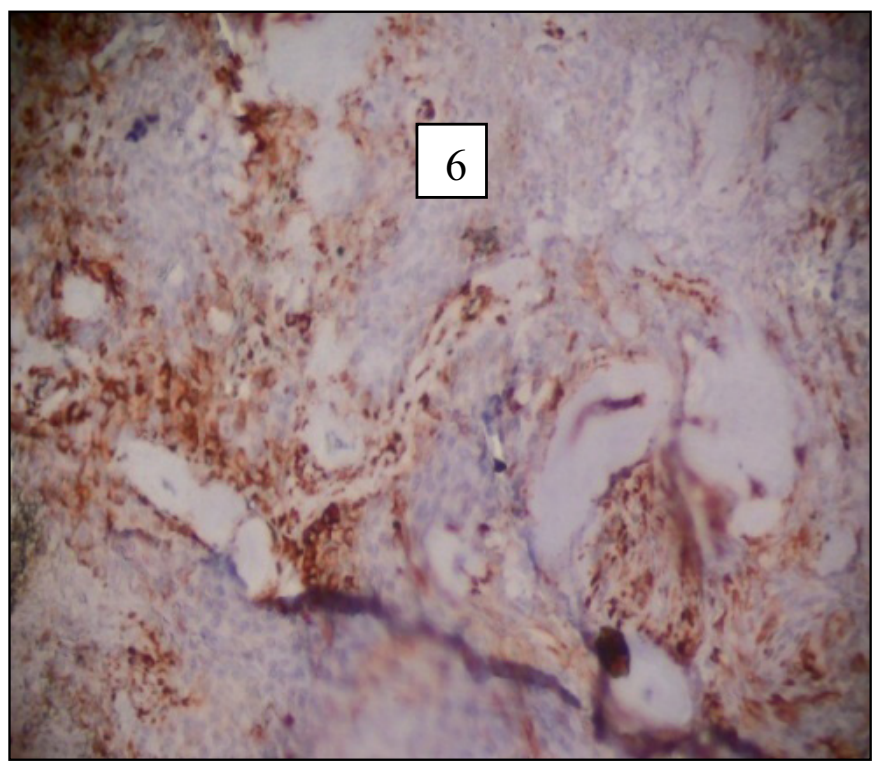

Fig. 6. Réaction positive aux CD34 cytoplasmiques des cellules endothéliales.

Fig. 6. Positive reaction at cytoplasmic CD34 of endothelial cells.

des limites car, chez ce patient, elle a orienté vers une lésion tumorale même si elle a permis de circonscrire les limites exactes de la tumeur. Plusieurs auteurs $[4,7,10]$ proposent l'IRM comme moyen d'orientation diagnostique tout en précisant que l'apport de l'imagerie est peu spécifique, tel qu'observé avec la tomodensitométrie, mais utile ; c'est une méthode prometteuse pour la détection surtout en cas de récidive voire pour le bilan topographique et morphologique de la lésion. Les lésions apparaissent hypo-intenses en pondération T1 et hyper-intenses en pondération T2. Les difficultés d'orientation diagnostique, du fait des localisations exceptionnelles, sont telles que seul l'examen histopathologique permet de poser le diagnostic. Cependant, la rareté de publication sur des localisations inhabituelles peut prêter à discussion sur ce diagnostic. Il est à noter qu'une seule autre localisation intrabuccale a déjà été rapportée, au niveau lingual en l'occurrence [8]. Ce constat est fait dans le cas présent où, même si les images histologiques étaient celles retrouvées dans la littérature, l'analyse immunohistochimique avait retrouvé une discordance dans le résultat en ce qui concerne la protéine S100. Le résultat aurait dû être négatif comme dans les localisations habituelles de cette tumeur, or c'était le contraire dans la localisation rapportée ici. Cette tumeur se subdivise en trois types, à savoir : la tumeur glomique classique, le glomangiome et le glomangiomyome. Certains auteurs [11-13] ont publié des cas exceptionnels de transformation maligne, on parle alors de glomangiosarcome. Le traitement est chirurgical et consiste en une exérèse large emportant la tumeur en monobloc [4], prévenant ainsi toute possibilité de récidive. 


\section{A S C L I N I Q U E}

\section{Références}

1. Duclos JY, Duffas 0, Deminière C, Darrouzet V, Stoll D. Glomangiome ou « tumeur glomique » des cavités nasales: à propos d'un nouveau cas et revue de la littérature. Rev Laryngol Otol Rhinol 2001;122:119-23.

2. Chu PG, Chang KL, Wu AY, Weiss LM. Nasal glomus tumors: report of two cases with emphasis on immunohistochemical features and differential diagnosis. Hum Pathol 1999;30(10):1259-61.

3. Miettinen M, Paal E, Lasota J, Sobin LH. Gastrointestinal glomus tumors: a clinicopathologic, immunohistochemical, and molecular genetic study of 32 cases. Am J Surg Pathol 2002;26(3):301-11.

4. Belkourchia E, Wahbi S, Bellarbi S, Moustaine R, Elmanouar M. Tumeur glomique à localisation inhabituelle. Rev Maroc Orthop Traumato 2005;24:44-5.

5. Weiss SW, Goldblum JR. Enzinger and Weiss's Soft tissue tumors. 4th ed. St Louis: Mosby 2001.

6. Wood WS, Dimmick JE. Multiple infiltrating glomus tumors in children. Cancer 1977;40 (4):1680-5.
7. Masson P. Le glomus neuro-myo-artériel des régions tactiles et ses tumeurs. Lyon Chir 1924;21:257-280.

8. Quesada R, Gonzalez-Lagunas J, Raspall G. Aggressive glomus tumor of tongue: report of a case. Med Oral 2004;9(4):350-4.

9. Boudghene FP, Gouny P, Tassart M, Callard P, Le Breton C, Vayssairat M. Subungual glomus tumor: Combined use of MRI and three-dimensional contrast MR angiography. J Magn Reson Imaging 1998;8(6):1326-8.

10. Theumann N, Baur A, Hauger O, Meyer P, Moushine E. Imagerie des tumeurs et des lésions d'apparence tumorale de la main. Rev Med Suisse 2005; 46 .

11. Muller A. Tumeur glomique extradigitale : une cause rare de névralgie. Ann Fr Anesth Réan 1997;1:61-3.

12. Perez de la Fuente T, Vega C, Gutierrez Pamacios A, Sanchez Lorenzo J, Gonzalez Sarasua J. Glomangiosarcoma of the hypothenar eminence: a case report. Rev Chir Main 2005;24 (3-4):199-02.

13. Aiba M, Hirayama A, Kuramochi S. Glomangiosarcoma in a glomus tumor: an immunohistochemical and ultrastructural study. Cancer 1988;61(7):1467-71. 